\title{
Cahiers de géographie du Québec
}

Meinig, D.W. (1971) Southwest : Three Peoples in Geographical

Change 1600-1970. Oxford University Press. 151 pages.

\section{Dean Louder}

Volume 18, numéro 44, 1974

URI : https://id.erudit.org/iderudit/021211ar

DOI : https://doi.org/10.7202/021211ar

Aller au sommaire du numéro

Éditeur(s)

Département de géographie de l'Université Laval

ISSN

0007-9766 (imprimé)

1708-8968 (numérique)

Découvrir la revue

Citer ce compte rendu

Louder, D. (1974). Compte rendu de [Meinig, D.W. (1971) Southwest : Three Peoples in Geographical Change 1600-1970. Oxford University Press. 151 pages.] Cahiers de géographie du Québec, 18(44), 404-405.

https://doi.org/10.7202/021211ar d'utilisation que vous pouvez consulter en ligne.

https://apropos.erudit.org/fr/usagers/politique-dutilisation/ 
MEINIG, D.W. (1971) Southwest: Three Peoples in Geographical Change 1600-1970. Oxford University Press. 151 pages.

Donald W. Meinig, celui qui nous a déjà donné les ouvrages importants sur de très diverses régions des États-Unis (The Great Columbia Plain, Imperial Texas et The Mormon Cultural Region), nous fournit dans ce travail un aperçu très révélateur et compréhensif d'une région américaine souvent négligée dans les manuels de géographie et certainement méconnue à la plupart des nord-américains. Southwest s'insère dans la série Historical Geography of North America publiée par les Presses de I'Université Oxford et dont l'éditeur est le professeur Andrew Clark de l'Université du Wisconsin.

Le Sud-Ouest, d'après Meinig, consiste surtout en deux États: l'Arizona et le Nouveau-Mexique. Une partie du sud du Colorado et du sud-est de I'Utah ainsi que la région d'El Paso au Texas en font également partie. C'est probablement la région des ÉtatsUnis, excluant l'Alaska, que l'on pourrait considérer comme étant la dernière frontière, les deux États principaux n'ayant accédé au statut d'état qu'en 1912. Les trois peuples en transition sont évidemment les premiers habitants, les Indiens, les Espagnols qui sont arrivés à partir de 1600 et les "Anglos" qui ne sont venus que vers la fin du dixneuvième siècle. Ces derniers ont été surtout attirés par la possibilité de faire l'élevage du bétail dans le cas des gens du Texas et la possibilité de pratiquer un type de culture irriguée dans le cas des Mormons.

Meinig décrit de façon générale les conflits qui ont eu lieu entre les Indiens et les Espagnols au commencement, mais il parle relativement peu des antagonismes qui se sont produits plus tard lors de la venue des "Anglos " et de l'implantation du chemin de fer. Meinig semble vouloir nous dire qu'au début le conflit existait mais qu'à travers le temps les trois cultures ont évolué ensemble pour enfin arriver à un état où elles jouissent chacune d'un rapport symbiotique. Ceci est peut-être vrai mais de telles conditions n'impliquent pas une égalité ou la justice de part et d'autre. Nulle part l'auteur ne fait mention de l'exploitation comme telle. II nous dit, par exemple, que les gens de tradition espagnole ou mexicaine sont moins bien rémunérés que leurs pendants Anglais et que les Indiens et les Mexicains ont traditionnellement été une source de "cheap labour", mais il ne donne pas d'explication et n'en tire aucun jugement de valeur. $\grave{A}$ ce sujet, il y a lieu de parler davantage de la région comme une exportatrice de main-d'œuvre de langue espagnole. Pourquoi faut-il que les vagues de migrants quittent le Sud-Ouest chaque année pour poursuivre une carrière de "migrant labourers"? Les réserves indiennes sont très souvent mentionnées mais les conditions de vie déplorables de celles-ci ne figurent pas dans le texte. On pourrait croire que tout va bien puisque "... the Navaho nation is now virtually a self-governing state within the States " (p. 115)

Deux chapitres traitent de la géographie sociale du Sud-Ouest, le premier portant sur la période 1900 et l'autre 1970, permettent de constater les changements qui sont survenus dans la région entre les deux périodes. Ces deux chapitres sont séparés de deux autres portant sur le développement économique et les tendances démographiques et politiques. Le rôle qu'a joué le gouvernement fédéral dans la région et l'importance de l'industrie du tourisme et de la villégiature sont évidents.

Les comparaisons urbaines faites par Meinig sont extrêmement intéressantes. Albuquerque, ville commerciale moderne et d'influence surtout Anglo est comparée à Santa $\mathrm{Fe}$, la vieille capitale et le centre de la culture hispanique dans la région. Tucson et El Paso qui partagent le même hinterland mais qui ont des fonctions très différentes, sont aussi comparées. On constate que la distance qui sépare Tucson de la frontière mexicaine lui donne un aspect hispanique plutôt artificiel par rapport à El Paso. La ville de Phoenix est un phénomène tout à fait spécial, un produit de la "losangelisation " de I'Arizona.

Ce livre montre que la géographie régionale peut être très analytique. Le cadre conceptuel est bien développé et c'est ce cadre qui permet à l'auteur d'aboutir à une excellente compréhension des ensembles régionaux. Meinig a le don de faire valoriser 
des régions qui semblent, à prime abord, moins importantes que les gros complexes urbains des Etats-Unis. Toute personne qui lira Southwest saisira mieux la dynamique de la nation américaine. Les drôles de toponymes qui apparaissent sur la carte des EtatsUnie prendront alors toute leur signification.

\author{
Dean LOUDER \\ Département de géographie \\ Université Laval
}

OfFICE DE PLANIFICATION ET DE DÉVELOPPEMENT DU QUÉBEC (O.P.D.Q.) (1973) Utilisation du sol des principales agglomérations du Québec. Québec, O.P.D.Q., Collection Études et Recherches. 1 atlas $46 \times 71 \mathrm{~cm}, 171$ planches. Distribué par le service des Relations publiques de l'Office de Planification et de Développement du Québec, Hôtel du Gouvernement, Québec.

L'établissement, sur une base comparative, de l'utilisation du sol des 62 agglomérations urbaines du Québec de plus de 4000 habitants a été réalisé durant l'été et l'automne 1971 par les départements de géographie de l'université Laval et de I'université de Montréal, sous la direction respective de monsieur Jean RAVENEAU et de madame Liane OTTMANN. Ce projet faisait partie d'un programme de recherches de l'Office de planification et de développement du Québec (OPDQ), placé sous la direction de monsieur Jean-Pierre MONTESINOS, et visant à constituer des indicateurs pour l'étude de l'armature urbaine du Québec, ceci afin de servir de matériau de base pour les recherches et l'élaboration de politiques futures d'urbanisation.

L'objectif principal du travail était de cartographier l'utilisation du sol des villes du Québec à l'aide de catégories comparables, à des échelles comparables et au même moment dans le temps, ceci afin de pouvoir établir des comparaisons entre les villes. La date choisie - l'été 1971 - permet d'effectuer des corrélations avec les données du recensement.

Les levés ont été effectué directement sur le terrain, avec report des données sur des cartes ou photographies aériennes dont l'échelle variait du 1/5000 au 1/20 000 . Les agglomérations ont été délimitées d'après l'extension de leur surface bâtie ; beaucoup d'entre elles débordent sur plusieurs municipalités. Pour la légende des cartes, six grandes catégories ont été retenues, certaines comprenant plusieurs sous-catégories ; 17 types d'utilisation du sol ont été retenus en tout :

R ZONES RÉSIDENTIELLES

$\mathrm{Ra}$-Zones de bungalows récents, à basse densité.

$\mathrm{Rb}$-Zones de résidences aisées, à basse densité.

Rc -Zones d'habitations séparées de type ancien, à moyenne densité.

Rd -Duplexes, Triplexes, maisons jumelées, maisons en rangées: densité moyenne à forte.

Rf -Tours à appartements à très forte densité.

$\mathrm{Rr}$ - Parcs de maisons mobiles.

Rs -Zones de chalets saisonniers.

C -ZONES COMMERCIALES, D'AFFAIRES ET DE SERVICES

Ca -Noyau central du centre-ville.

$\mathrm{Cb}$-Centres d'achats.

Cc -Zones et bâtiments commerciaux secondaires.

E ZONES INSTITUTIONNELLES

Ea -Espaces verts ouverts à des fins récréationnelles.

Eb -Bâtiments institutionnels.

U -ESPACES INDUSTRIELS

$U$-Bâtiments industriels et leurs dépendances.

Um -Vastes espaces liés aux industries ou à l'extraction. 